\title{
Molecule Detection With Graphene Dimer
}

Nanoantenna.

\section{Supporting Information.}

\author{
François Aguillon, Dana Codruta Marinica, and Andrei G. Borisov \\ Institut des Sciences Moléculaires d'Orsay, UMR 8214, CNRS, Université Peris-Saclay, \\ Bâtiment 520, 91405 Orsay Cedex, France
}

\begin{abstract}
In this Supporting Information we detail the theoretical approach used in the main paper. We address the following issues:

- The time dependent tight binding description of the electron the density dynamics of graphene;

- The calculation of the ground state and of the optical response of the graphene nanoantennas;

- The calculation of the optical response of the nanoantennas functionalised with $\mathrm{CO}$ and $\mathrm{N}_{2}$ molecules.
\end{abstract}




\section{Bare nanoantenna. The time-dependent tight binding ap- proach to electron density dynamics.}

The time dependent approach for the calculation of the electron density evolution in response to an external perturbation is introduced with an example of the individual graphene nanoantenna. The optical response of the nanoantenna + molecule system is addressed in the following sections.

\section{The time-dependent tight binding approach}

The time evolution of the electronic density, $n(\vec{r}, t)$, of the nanoantenna subjected to the external electromagnetic pulse with electric field $\vec{E}_{\mathrm{IR}}(t)$ can be expressed using the formalism of the time-dependent density functional theory (TDDFT). Within the Kohn-Sham (KS) scheme, $n(\vec{r}, t)$ is represented via the sum over occupied KS orbitals $n(\vec{r}, t)=2 \sum_{j \in \text { occ }}\left|\psi_{j}(\vec{r}, t)\right|^{2}$, where 2 is the spin statistical factor. The occupied (occ) KS orbitals $\psi_{j}(\vec{r}, t)$ evolve in time according to

$$
i \partial_{t} \psi_{j}(\vec{r}, t)=\left\{\hat{H}^{0}+V(\triangle n ; \vec{r}, t)+V_{\mathrm{ext}}(\vec{r}, t)\right\} \psi_{j}(\vec{r}, t)
$$

where $\hat{H}^{0}$ is the Hamiltonian of the ground-state graphene nanoparticle. $V(\triangle n ; \vec{r}, t)$ is the change of the electron-graphene interaction because the electron density evolved from that of the ground state, $n_{0}(\vec{r})$. The induced density is defined as $\triangle n(\vec{r}, t)=n(\vec{r}, t)-n_{0}(\vec{r})$. Finally, the third term in curly brackets stands for the potential owing to an external perturbation (e.g. $V_{\text {ext }}(\vec{r}, t)=\vec{r} \vec{E}_{\mathrm{IR}}(t)$ for the incident IR laser pulse with electric field $\left.\vec{E}_{\mathrm{IR}}(t)\right)$.

The relevant Dirac plasmon mode of graphene nanoparticles corresponds to the electronic excitations within the $2 \mathrm{D} \pi$-band close to the $K$ point in the reciprocal space. In this situation the tight binding (TB) model of graphene nanoparticles can be applied in order to efficiently tackle relatively large objects. ${ }^{1|2|}$ Despite its simplicity the TB model has been used to quantitatively predict variety of linear and nonlinear effects in graphene plasmonics. $2-6$ 
The KS wave functions $\psi_{j}(\vec{r}, t)$ are projected on the basis of the out-of-plane $2 p$-orbitals of the carbon atoms at $\vec{r}_{\ell}$ lattice sites: $\psi_{j}(\vec{r}, t)=\sum_{\ell} \Psi_{\ell}(j, t) \varphi_{2 p}\left(\vec{r}-\vec{r}_{\ell}\right)$.

Thus, within the TB representation $\psi_{j}(\vec{r}, t) \rightarrow \boldsymbol{\Psi}(j, t)$, where $\boldsymbol{\Psi}(j, t)$ is the column formed by the $\Psi_{\ell}(j, t)$ coefficients with $\ell=1, \ldots, N$ ( $N$ is the number of carbon atoms). Eq. 1 is then cast in the form

$$
i \partial_{t} \Psi(j, t)=\left\{\mathbb{H}^{0}+\mathbb{V}(t)+\mathbb{V}^{\mathrm{ext}}(t)\right\} \boldsymbol{\Psi}(j, t),
$$

where index $j$ runs over all initially occupied KS orbitals, $\mathbb{H}^{0}$ is the $N \times N$ ground-state Hamiltonian matrix, and $\mathbb{V}(t)$ is the $N \times N$ diagonal matrix of the induced potential resulting from the evolution of the density of the system from that of the ground state. Finally $\mathbb{V}_{\text {ext }}(t)$ is the $N \times N$ diagonal matrix of the potential produced by an external perturbation.

Following Ref., ${ }^{2} \mathbb{V}(t)$ is given by

$$
\mathbb{V}_{\ell \ell^{\prime}}(t)=\left(\sum_{k \neq \ell} \frac{\Delta q_{k}(t)}{\left|\overrightarrow{r_{\ell}}-\overrightarrow{r_{k}}\right|}+0.58 \Delta q_{\ell}(t)\right) \delta_{\ell \ell^{\prime}}
$$

where the induced charge variation of the carbon atom sites can be found from

$$
\Delta q_{\ell}(t)=2 \sum_{j}\left\{\left|\Psi_{\ell}(j, t)\right|^{2}-\left|\Psi_{\ell}^{0}(j)\right|^{2}\right\} .
$$

The ground-state KS orbitals $\Psi_{\ell}^{0}(j)$ are the eigenstates of the $\mathbb{H}^{0}$ Hamiltonian. The timedependent tight binding (TDTB) theory thus neglects the variation of the exchange-correlation potential and only takes into account the change of the Hartree potential associated with density evolution. In the case of the weak perturbation and linear response, the TDTB model outlined here is the time-domain analogue of the frequency-domain random phase approximation (RPA) description of graphene plasmons. ${ }^{20}$ It is well justified because of the nature of the plasmon oscillations in the system, where conduction electrons dynamically screen the induced charges. In absence of the Lindblad relaxation operators ${ }^{[7]}$ the electron 
density dynamics described with time dependent equations Eq. 2 is equivalent to the density matrix description used in Ref.1

The time dependent equations Eqs. 2 are solved applying the short-time Lanczos propagation scheme ${ }^{8 \mid 9}$ with nanoantenna initially in the ground state, $\Psi_{\ell}(j, t=0)=\Psi_{\ell}^{0}(j)$.

\section{Optical Response}

The choice of the external perturbation $\mathbb{V}^{\text {ext }}(t)$ allows to calculate the optical response of the nanoantenna and depends on the problem at hand.

- For the calculation of the density dynamics induced by an incident laser pulse the external potential is taken in the form $\mathbb{V}_{\ell \ell^{\prime}}^{\text {ext }}(t)=\vec{r}_{\ell} \cdot \vec{E}_{\mathrm{IR}}(t) \delta_{\ell \ell^{\prime}}$.

- For the calculations of the absorption spectra the impulsive perturbation $\vec{E}_{\mathrm{IR}}(t)=$ $\kappa \hat{e}_{x} \delta(t)$ is used, where $\kappa$ is a small positive number, and $\hat{e}_{x}$ is the unit length vector along the dimer axis defined here as $x$-axis. Typically we use $\kappa=10^{-4}$, and we have explicitly checked that for this choice of the field amplitude the linear response holds. The absorption cross section is then obtained from $\sigma=\frac{4 \pi \omega}{c} \operatorname{Im}\left\{\left(\overleftrightarrow{\alpha}_{\mathrm{Gr}}(\omega)\right)_{x x}\right\}=$ $\frac{4 \pi \omega}{c} \operatorname{Im}\left\{\frac{1}{\kappa} \hat{e}_{x} \cdot \vec{P}_{\mathrm{Gr}}(\omega)\right\}$, where $\operatorname{Im}\{Z\}$ stands for the imaginary part of the complex number $Z,\left(\overleftrightarrow{\alpha}_{\mathrm{Gr}}(\omega)\right)_{x x}$ is the diagonal element of the graphene polarizability tensor, and $\vec{P}_{\mathrm{Gr}}(\omega)$ is the dipole induced at graphene nanostructure. It is obtained from the TDTB result $\vec{P}_{\mathrm{Gr}}(t)$ using the time-to-frequency Fourier transform. The same calculation also yields the field-field response $\hat{\mathbb{F}}(\vec{R}, \omega)$ defined with relation $\vec{E}_{\text {ind }}(\vec{R}, \omega)=$ $\hat{\mathbb{F}}(\vec{R}, \omega) \vec{E}_{\mathrm{IR}}(\omega)$. Here $\vec{E}_{\text {ind }}(\vec{R}, \omega)$ is the induced field produced at position $\vec{R}$ by graphene nanostructure in response to the incident field $\vec{E}_{\mathrm{IR}}(\omega)$.

- For the calculations of the Green's dyadic $\hat{\mathbb{G}}\left(\vec{R}, \overrightarrow{R^{\prime}}, \omega\right)$ we proceed as follows. In Eq. 2 the external potential is set as $\mathbb{V}_{\ell \ell^{\prime}}^{\mathrm{ext}}(t)=\frac{\hat{e}_{x, y, z} \cdot\left(\vec{R}-\vec{r}_{\ell}\right)}{\left|\vec{R}-\vec{r}_{\ell}\right|^{3}} \kappa \delta(t) \delta_{\ell \ell^{\prime}}$ i.e. it is given by the potential created by a point dipole $\kappa \hat{e}_{i},(i=x, y, z)$ located in plasmonic gap at position $\vec{R}$. Typically we use $\kappa=10$, and we have explicitly checked that for this choice of the 
dipole amplitude the linear response holds. Then, the $\hat{\mathbb{G}}\left(\vec{R}, \vec{R}^{\prime}, \omega\right)$ can be obtained with TDTB from the electric field created at position $\overrightarrow{R^{\prime}}$ by graphene nanostructure. From the same calculation we obtain, $\hat{\mathbb{P}}(\vec{R}, \omega)$, the polarization response of the graphene antenna to the unitary point dipole $\hat{e}_{i},(i=x, y, z)$. The polarization response is

defined with $\vec{P}_{\mathrm{Gr}}(\omega)=\hat{\mathbb{P}}(\vec{R}, \omega) \vec{P}_{\mathrm{Mol}}$, where $\vec{P}_{\mathrm{Gr}}$ is the dipole induced on graphene nanoantenna by the point dipole $\vec{P}_{\text {Mol }}$ located at $\vec{R}$.

The TDTB approach based on the adiabatic Hartree potentials does not allow a complete account of the decay and dephasing of the plasmon modes because of the interaction with phonons as well as because of the intrinsic multi-electron mechanisms. ${ }^{10}$ Thus, the plasmon peak appears as a group of sharp spectral features owing to the particle-hole excitations. This is a well-known issue in the TDDFT or RPA calculations. ${ }^{2 / 13}$ An artificial broadening $\gamma$ of the spectral features is then introduced. It mimics various decay and dephasing channels not included into the theory, and it results in a smooth plasmon peak in the absorption spectra as shown in the main paper. In practice, the time-to frequency Fourier analysis is performed with $e^{i(\omega+i \gamma / 2) t}$ exponential instead of $e^{i \omega t}$. We use $\gamma=30 \mathrm{meV}$. In the case of circular nanoparticles, the resulting width of the plasmon resonance equals to $\approx 2 \gamma$. This illustrates the importance of the plasmon decay via interaction with nanoflake edges accounted for in our approach. $\frac{214}{214}$ For triangular shape nanoparticles the edge effects are small. The width of the plasmon resonance is given by $\gamma$ which is taken here within the range of the typical values reported for graphene nanostructures. $2117 / 18$

\section{The ground state calculation}

The initial conditions for the TDTB calculations of the density dynamics correspond to the graphene nanoantena being in the ground state. Thus, the ground-state properties of the system have to be calculated prior to the time-propagation. The ground-state Hamiltonian 
of the graphene antenna is given by

$$
\mathbb{H}^{0}=\mathbb{H}^{\mathrm{Gr}}+\mathbb{V}^{0}
$$

where $\mathbb{H}_{\ell \ell^{\prime}}^{\mathrm{Gr}}=g$ for atomic positions $\ell^{\prime}$ corresponding to the nearest neighbors of the atom $\ell$, otherwise $\mathbb{H}_{\ell \ell^{\prime}}^{\mathrm{Gr}}=0$. The hopping integral $g=-2.8 \mathrm{eV} \cdot 19$ The potential matrix $\mathbb{V}^{0}$ is diagonal with $V_{\ell \ell}^{0}=\sum_{k \neq \ell} \frac{\Delta q_{k}^{0}}{\left|\overrightarrow{\vec{r}_{\ell}}-\vec{r}_{k}\right|}+0.58 \Delta q_{\ell}^{0} \cdot \frac{112 \mid}{}$ The charge induced at the carbon site $k$ in this case is given by $\Delta q_{k}^{0}=2 \sum_{j}\left|\Psi_{k}^{0}(j)\right|^{2}-1$. To determine the orbitals $\Psi^{0}(j)$ given by the vector of the $\Psi_{f}^{0}(j)$-coefficients an iterative procedure is applied. It is started with eigenstates $\Psi^{\mathrm{Gr}}(j)$ of the $\mathbb{H}^{\mathrm{Gr}}$ matrix $\left(\mathbb{H}^{\mathrm{Gr}} \boldsymbol{\Psi}^{\mathrm{Gr}}(j)=E_{j}^{\mathrm{Gr}} \boldsymbol{\Psi}^{\mathrm{Gr}}(j)\right)$ corresponding to the case of the uniform charge doping. The orbitals $\Psi^{\mathrm{Gr}}(j)$ are double occupied for $E_{j}^{\mathrm{Gr}} \leq E_{F}$, and unoccupied for $E_{j}^{\mathrm{Gr}}>E_{F}$. Then, the $\mathbb{V}^{0}$ potential is calculated and iterations are continued with $\mathbb{H}^{0}$ matrix diagonalization.

In the situation where one is interested only in the linear response properties of the system and in the low doping regime, the calculation of the ground state can be essentially simplified assuming uniform doping $\boldsymbol{\Psi}^{0}(j)=\boldsymbol{\Psi}^{\mathrm{Gr}}(j)$. Here, the boundary effects are neglected. The validity of this approximation used in the present work has been discussed in Ref. ${ }^{20}$ and in Ref. ${ }^{21}$

It is worth noting that for the ground state of the graphene nanoflakes in the compound molecule + graphene system we use the same initial conditions as for the free-standing graphene nanoantenna. We have explicitly tested that e.g. the effect of the perturbation of the ground state by the small $(0.125 \mathrm{D})$ permanent dipole of $\mathrm{CO}$ molecule ${ }^{222}$ is negligible as far as the absorption spectrum of the compound system is concerned. This issue however has to be taken with care for charged analyte, molecules with larger dipole moments, or as far as nonlinear response is concerned..$^{5}$ 


\section{Nanoantenna + CO molecule}

The absorption cross-section of the compound system: graphene nanoantenna + CO molecule is obtained from the imaginary part of the total dipole $\overrightarrow{\mathcal{P}}=\vec{P}_{\mathrm{Gr}}+\vec{P}_{\text {Mol }}$. For the incident field $\vec{E}_{\mathrm{IR}}(\omega)$, using the notations introduced above, the dipole induced on graphene nanoantenna, $\vec{P}_{\mathrm{Gr}}$, and molecular dipole, $\vec{P}_{\mathrm{Mol}}$, can be obtained from:

$$
\begin{aligned}
\vec{P}_{\mathrm{Gr}}(\omega) & =\overleftrightarrow{\alpha}_{\mathrm{Gr}}(\omega) \vec{E}_{\mathrm{IR}}+\hat{\mathbb{P}}(\vec{R}, \omega) \vec{P}_{\mathrm{Mol}}(\omega) \\
\vec{P}_{\mathrm{Mol}}(\omega) & =\overleftrightarrow{\alpha}_{\mathrm{Mol}}(\omega)\{\hat{\mathbb{I}}+\hat{\mathbb{F}}(\vec{R}, \omega)\} \vec{E}_{\mathrm{IR}}+\overleftrightarrow{\alpha}_{\mathrm{Mol}}(\omega) \hat{\mathbb{G}}(\vec{R}, \vec{R}, \omega) \vec{P}_{\mathrm{Mol}}(\omega)
\end{aligned}
$$

where $\hat{\mathbb{I}}$ is the identity tensor, and $\overleftrightarrow{\alpha}_{\operatorname{Mol}}(\omega)$ is a molecular polarisability tensor. Using the second equation,

$$
\vec{P}_{\mathrm{Mol}}(\omega)=\frac{1}{\hat{\mathbb{I}}-\overleftrightarrow{\alpha}_{\mathrm{Mol}}(\omega) \hat{\mathbb{G}}(\vec{R}, \vec{R}, \omega)} \overleftrightarrow{\alpha}_{\mathrm{Mol}}(\omega)\{\hat{\mathbb{I}}+\hat{\mathbb{F}}(\vec{R}, \omega)\} \vec{E}_{\mathrm{IR}}
$$

For the CO molecule the $\overleftrightarrow{\alpha}_{\text {Mol }}(\omega)$ is given by

$$
\overleftrightarrow{\alpha}_{\mathrm{Mol}}(\omega)=\hat{e}_{M}\left[\frac{2 \Omega \mu^{2}}{\Omega^{2}-\omega^{2}-i \omega \eta}\right] \hat{e}_{M}
$$

where $\hat{e}_{M}$ is the unit length vector in direction of molecular axis. The excitation energy, $\Omega=263 \mathrm{meV}^{23}$ is given by the difference between the energies of the $v=0$ and $v=1$ vibrational states, and $\eta$ is the width of molecular resonance. The coupling matrix element $\mu$ between vibrational wave functions $\varphi_{v}(\xi)$ ( $\xi$ is the CO bond length) is given by $\mu=\frac{d P_{\mathrm{CO}}}{d \xi}\left\langle\varphi_{v=0}(\xi)|\xi| \varphi_{v=1}(\xi)\right\rangle$. Here, $P_{\mathrm{CO}}$ is the $\xi$-dependent electric dipole of the freestanding CO molecule obtained with ab initio quantum chemistry calculations. $\frac{23}{2}$ The derivative $\frac{d P_{\mathrm{CO}}(0)}{d \xi}$ is evaluated at the equilibrium position $\xi=0$. The real valued $\varphi_{v}(\xi)$ are obtained from the stationary Schrodinger equation describing molecular stretching motion in the ab initio potential. $\underline{23}$

Eq. 7 takes a particularly simple form for the case of the $x$-polarised incident field and 
the CO molecule aligned along the dimer axis. Only the $x$-components of the fields and polarisations are then non-zero, and, considering $e^{-i \omega t}$ dependence of the fields, we obtain close to the resonance $(\omega \sim \Omega)$.

$$
P_{\mathrm{Mol}}(\omega)=\frac{\mu^{2}[1+F(\vec{R}, \omega)]}{\Omega-\omega-i \eta / 2-\mu^{2} G(\vec{R}, \vec{R}, \omega)} E_{\mathrm{IR}}
$$

Because of the presence of the plasmonic nanostructure, the molecular absorption resonance appears shifted in energy and its lifetime is reduced. This Lamb shift and Purcell effect (given respectively by the real $(R e)$ and imaginary $(I m)$ parts of the self-interaction $\mu^{2} G(\vec{R}, \vec{R}, \omega)$ are well studied for excitons in plasmonic environments. 24 31 Here we discuss the energy shift and increased decay rate for molecular vibrational excitation. $\stackrel{32+34}{\text { Interestingly, at }}$ resonance $\omega=\Omega-\operatorname{Re}\left\{\mu^{2} G(\vec{R}, \vec{R}, \omega)\right\}$, if $\eta$ can be neglected in Eq. 9 one obtains $P_{\text {Mol }}(\omega)=$ $i \frac{1+F(\vec{R}, \omega)}{\operatorname{Im}\{G(\vec{R}, \vec{R}, \omega)\}}$ independent of molecular characteristics. As a consequence, the dipole induced at graphene nanoantenna in response to $P_{\mathrm{Mol}}$ is also independent of molecular characteristics. So is the molecule induced feature in the absorption cross-section.

\section{Nanoantenna $+\mathrm{N}_{2}$ molecule}

The $\mathrm{N}_{2}$ molecule does not possess a dipole moment so that

- The absorption cross section is given by the electric dipole induced on graphene nanoantenna.

- The vibrational excitation of the molecule proceeds via quadrupolar coupling owing to the inhomogeneity of plasmonic near field.

The potential energy of the quadrupole in the electric field is given by $V=-(\overleftrightarrow{Q} \vec{\nabla}) \cdot \vec{E}$. The electric quadrupole tensor $\overleftrightarrow{Q}$ is defined as $Q_{i j}=\frac{1}{2} \iiint d^{3} \vec{r} \rho(\vec{r})\left(r_{i} r_{j}-\frac{1}{3} r^{2}\right)$, where $\rho(\vec{r})$ is the molecular charge density, and $r_{j},(j=x, y, z)$ stands for the projection of the position vector $\vec{r}$ on the corresponding axis. For the linear molecule such as $\mathrm{N}_{2}$ it is convenient to 
use the coordinate frame with $z$-axis along the molecular bond. Only the diagonal elements of $\overleftrightarrow{Q}$ are thus nonzero with $2 Q_{x x}=2 Q_{y y}=-Q_{z z}$ so that the interaction potential can be cast in the form

$$
V=-Q_{z z}\left(\frac{d E_{z}}{d z}-\frac{1}{2} \frac{d E_{x}}{d x}-\frac{1}{2} \frac{d E_{y}}{d y}\right)
$$

To obtain the plasmon-vibration quadrupolar coupling, the bond length $\xi$-dependent molecular quadrupole $\overleftrightarrow{Q}^{N_{2}}(\xi)$ is calculated using the ab initio quantum chemistry software. $\frac{35}{35}$ Close to the equilibrium position $(\xi=0)$ the $Q_{z z}^{\mathrm{N}_{2}}(\xi)$ can be developed in Taylor series. Retaining only the first two terms: $Q_{z z}^{\mathrm{N}_{2}}(\xi)=Q_{z z}^{\mathrm{N}_{2}}(0)+\frac{d Q_{z z}^{\mathrm{N}_{2}}}{d \xi}(0) \xi$. The quadrupolar moment $Q_{z z}$ of the molecule associated with $v=0 \rightarrow v=1$ vibrational excitation in the inhomogeneous near fields can be then found from

$$
Q_{z z}(\omega)=\frac{2 \Omega \mu_{Q}^{2}}{\Omega^{2}-\omega^{2}-i \omega \eta}\left(\frac{d E_{z}(\vec{R}, \omega)}{d z}-\frac{1}{2} \frac{d E_{x}(\vec{R}, \omega)}{d x}-\frac{1}{2} \frac{d E_{y}(\vec{R}, \omega)}{d y}\right)
$$

The transition matrix element $\mu_{Q}$ is given by

$$
\mu_{Q}=\frac{d Q_{z z}^{\mathrm{N}_{2}}(0)}{d \xi}\left\langle\varphi_{v=0}(\xi)|\xi| \varphi_{v=1}(\xi)\right\rangle
$$

where the derivative $\frac{d Q_{z z}^{N_{2}}}{d \xi}$ is taken at the equilibrium position $\xi=0$. The $\mathrm{N}_{2}$ vibrational excitation energy $\Omega=296 \mathrm{meV}$ and the vibrational wave functions $\varphi_{v}(\xi)$ are obtained from the quantum chemistry calculations. .35

The self-consistent near field of the graphene nanostructure at the position of the molecule accounts for response of the nanoantenna to the (i) incident field, and to the (ii) presence of molecular quadrupole

$$
\vec{E}(\vec{R}, \omega)=\hat{\mathbb{F}}(\vec{R}, \omega) \vec{E}_{\mathrm{IR}}+\hat{\mathbb{G}}_{\mathrm{Q}}(\vec{R}, \vec{R}, \omega) \overleftrightarrow{Q}(\omega)
$$

where $\hat{\mathbb{G}}_{\mathrm{Q}}(\vec{R}, \vec{R}, \omega)$ is analogous of the Green's dyadic for the dipole excitation. It is obtained from the TDTB calculations where the external potential acting on the nanoantenna, 
$\mathbb{V}^{\text {ext }}(t)$, is given by the impulsive potential created by the probe quadrupole located at $\vec{R}$.

Along with $\hat{\mathbb{G}}_{\mathrm{Q}}(\vec{R}, \vec{R}, \omega)$ this calculation also yields $\hat{\mathbb{P}}_{\mathrm{Q}}(\vec{R}, \omega)$ - the polarisation response of the nanoantenna to the quadrupole located at $\vec{R}$. Finally the dipole induced on graphene nanostructure, $\vec{P}_{\mathrm{Gr}}$ can be expressed as

$$
\vec{P}_{\mathrm{Gr}}(\omega)=\overleftrightarrow{\alpha}_{\mathrm{Gr}}(\omega) \vec{E}_{\mathrm{IR}}+\hat{\mathbb{P}}_{\mathrm{Q}}(\vec{R}, \omega) \overleftrightarrow{Q}(\omega)
$$

The equations Eq.11. Eq. 13, and Eq.14 are then solved self-consistently and absorption spectra of compound nanostructure are obtained.

\section{References}

(1) Cox, J. D.; Silveiro, I.; García de Abajo, F. J. Quantum Effects in the Nonlinear Response of Graphene Plasmons. ACS Nano 2016, 10, 1995-2003.

(2) Thongrattanasiri, S.; Manjavacas, A.; García de Abajo, F. J. Quantum Finite-Size Effects in Graphene Plasmons. ACS Nano 2012, 6, 1766-1775.

(3) Thongrattanasiri, S.; Manjavacas, A.; Nordlander, P.; de Abajo, F. J. G. Quantum junction plasmons in graphene dimers. Laser and Photonics Reviews 2013, 7, 297-302.

(4) Thongrattanasiri, S.; García de Abajo, F. J. Optical Field Enhancement by Strong Plasmon Interaction in Graphene Nanostructures. Phys. Rev. Lett. 2013, 110, 187401.

(5) Yu, R.; Cox, J. D.; de Abajo, F. J. G. Nonlinear Plasmonic Sensing with Nanographene. Phys. Rev. Lett. 2016, 11\%, 123904.

(6) Cox, J. D.; García de Abajo, F. J. Nonlinear Graphene Nanoplasmonics. Accounts of Chemical Research 2019, 52, 2536-2547.

(7) Alicki, R.; Kosloff, R. In Thermodynamics in the Quantum Regime: Fundamental As- 
pects and New Directions; Binder, F., Correa, L. A., Gogolin, C., Anders, J., Adesso, G., Eds.; Springer International Publishing: Cham, 2018; pp 1-33.

(8) Park, T. J.; Light, J. C. Unitary quantum time evolution by iterative Lanczos reduction. The Journal of Chemical Physics 1986, 85, 5870-5876.

(9) Leforestier, C.; Bisseling, R.; Cerjan, C.; Feit, M.; Friesner, R.; Guldberg, A.; Hammerich, A.; Jolicard, G.; Karrlein, W.; Meyer, H.-D.; Lipkin, N.; Roncero, O.; Kosloff, R. A comparison of different propagation schemes for the time dependent Schrödinger equation. Journal of Computational Physics 1991, 94, 59-80.

(10) Escartín, J. M.; Vincendon, M.; Romaniello, P.; Dinh, P. M.; Reinhard, P.-G.; Suraud, E. Towards time-dependent current-density-functional theory in the non-linear regime. The Journal of Chemical Physics 2015, 142, 084118.

(11) Wijewardane, H. O.; Ullrich, C. A. Time-Dependent Kohn-Sham Theory with Memory. Phys. Rev. Lett. 2005, 95, 086401.

(12) Vignale, G.; Kohn, W. Current-Dependent Exchange-Correlation Potential for Dynamical Linear Response Theory. Phys. Rev. Lett. 1996, 77, 2037-2040.

(13) Varas, A.; García-González, P.; Feist, J.; García-Vidal, F.; Rubio, A. Quantum plasmonics: from jellium models to ab initio calculations. Nanophotonics 01 Aug. 2016, $5,409-426$.

(14) Christensen, T.; Wang, W.; Jauho, A.-P.; Wubs, M.; Mortensen, N. A. Classical and quantum plasmonics in graphene nanodisks: Role of edge states. Phys. Rev. B 2014, 90, 241414 .

(15) Marques, M. A. L.; Gross, E. K. U. Time-dependent density functional theory. Annu. Rev. Phys. Chem. 2004, 55, 427-455. 
(16) Burke, K.; Werschnik, J.; Gross, E. K. U. Time-dependent density functional theory: Past, present, and future. J. Chem. Phys. 2005, 123, 062206.

(17) Fang, Z.; Wang, Y.; Schlather, A. E.; Liu, Z.; Ajayan, P. M.; García de Abajo, F. J.; Nordlander, P.; Zhu, X.; Halas, N. J. Active Tunable Absorption Enhancement with Graphene Nanodisk Arrays. Nano Letters 2014, 14, 299-304.

(18) Rodrigo, D.; Limaj, O.; Janner, D.; Etezadi, D.; García de Abajo, F. J.; Pruneri, V.; Altug, H. Mid-infrared plasmonic biosensing with graphene. Science 2015, 349, 165168.

(19) Castro Neto, A. H.; Guinea, F.; Peres, N. M. R.; Novoselov, K. S.; Geim, A. K. The electronic properties of graphene. Rev. Mod. Phys. 2009, 81, 109-162.

(20) Thongrattanasiri, S.; Silveiro, I.; Javier García de Abajo, F. Plasmons in electrostatically doped graphene. Applied Physics Letters 2012, 100, 201105.

(21) Silveiro, I.; Javier García de Abajo, F. Plasmons in inhomogeneously doped neutral and charged graphene nanodisks. Applied Physics Letters 2014, 104, 131103.

(22) Scuseria, G. E.; Miller, M. D.; Jensen, F.; Geertsen, J. The dipole moment of carbon monoxide. The Journal of Chemical Physics 1991, 94, 6660-6663.

(23) Langhoff, S. R.; Bauschlicher, C. W. Global dipole moment function for the $X^{1} \Sigma^{+}$ ground state of CO. The Journal of Chemical Physics 1995, 102, 5220-5225.

(24) Andreussi, O.; Corni, S.; Mennucci, B.; Tomasi, J. Radiative and nonradiative decay rates of a molecule close to a metal particle of complex shape. J. Chem. Phys. 2004, 121, 10190-10202.

(25) Bouchet, D.; Carminati, R. Quantum dipole emitters in structured environments: a scattering approach: tutorial. J. Opt. Soc. Am. A 2019, 36, 186-195. 
(26) Anger, P.; Bharadwaj, P.; Novotny, L. Enhancement and Quenching of Single-Molecule Fluorescence. Phys. Rev. Lett. 2006, 96, 113002.

(27) Van Vlack, C.; Kristensen, P. T.; Hughes, S. Spontaneous emission spectra and quantum light-matter interactions from a strongly coupled quantum dot metal-nanoparticle system. Phys. Rev. B 2012, 85, 075303.

(28) Delga, A.; Feist, J.; Bravo-Abad, J.; Garcia-Vidal, F. J. Quantum emitters near a metal nanoparticle: strong coupling and quenching. Phys. Rev. Lett. 2014, 112, 253601.

(29) Akselrod, G. M.; Argyropoulos, C.; Hoang, T. B.; Ciracì, C.; Fang, C.; Huang, J.; Smith, D. R.; Mikkelsen, M. H. Probing the mechanisms of large Purcell enhancement in plasmonic nanoantennas. Nature Photonics 2014, 8, 835.

(30) Carminati, R.; Cazé, A.; Cao, D.; Peragut, F.; Krachmalnicoff, V.; Pierrat, R.; Wilde, Y. D. Electromagnetic density of states in complex plasmonic systems. Sur. Sci. Rep. 2015, 70, 1-41.

(31) Zhang, C.; Chen, B.-Q.; Li, Z.-Y. Optical origin of subnanometer resolution in tipenhanced raman mapping. J. Phys. Chem. C 2015, 119, 11858-11871.

(32) Ford, G.; Weber, W. Electromagnetic interactions of molecules with metal surfaces. Phys. Rep. 1984, 113, 195-287.

(33) Thomas, A.; Lethuillier-Karl, L.; Nagarajan, K.; Vergauwe, R. M. A.; George, J.; Chervy, T.; Shalabney, A.; Devaux, E.; Genet, C.; Moran, J.; Ebbesen, T. W. Tilting a ground-state reactivity landscape by vibrational strong coupling. Science $\mathbf{2 0 1 9}$, 363, 615-619.

(34) Galego, J.; Climent, C.; Garcia-Vidal, F. J.; Feist, J. Cavity Casimir-Polder Forces and Their Effects in Ground-State Chemical Reactivity. Phys. Rev. X 2019, 9, 021057.

(35) Frisch, M. J. et al. Gaussian 16 Revision C.01. 2016; Gaussian Inc. Wallingford CT. 